\title{
Scanning Tunneling Microscopy and Spectroscopy of Peptide-Wrapped Single- Walled Carbon Nanotubes
}

\author{
I. H. Musselman ${ }^{* * *}$, V. Z. Poenitzsch ${ }^{*}$, and G. R. Dieckmann ${ }^{* * *}$ \\ *Department of Chemistry, University of Texas at Dallas, Richardson, TX 75083 \\ ${ }^{* *}$ NanoTech Institute, University of Texas at Dallas, Richardson, TX 75083
}

Changes to the electronic structure of single-walled carbon nanotubes (SWNT) through functionalization are of fundamental importance to potential SWNT-based molecular devices and sensors. Noncovalent functionalization offers the promise of introducing various functional groups without perturbing the $\pi$-system and electronic structure of SWNTs. We have previously demonstrated that a designed polypeptide, denoted nano-1, coats and debundles SWNTs and promotes their assembly into novel hierarchical structures [1, 2]. Nano-1 folds into an amphiphilic $\alpha$-helix, in which the apolar residues occupy one face of the helix and more polar residues form the other face. The hydrophobic face was designed to noncovalently interact with the SWNT, and the hydrophilic face was designed to interact with water. In this study, we examine the effect of the noncovalent protein functionalization on the electronic properties of SWNTs using scanning tunneling microscopy (STM) and scanning tunneling spectroscopy (STS).

Nano-1/SWNT dispersions [2] were spun cast onto $\mathrm{Au}(111)$-coated mica substrates. Atomic force microscopy (AFM) images (Digital Instruments Nanoscope III Multimode Scanning Probe Microscope operated in TappingMode ${ }^{T M}$ ) of nano-1/SWNT dispersions and diameter distribution, determined from AFM height measurements, confirmed a suitable density of individual peptidewrapped SWNTs for further STM and STS analyses (Fig. 1). STM measurements were performed using a Digital Instruments Nanoscope III STM operated at room temperature under ambient conditions with Pt/Ir tips, current setpoints of 400 to $600 \mathrm{pA}$, and bias voltages of 600 to $800 \mathrm{mV}$. Overall, STM images showed that SWNTs were efficiently coated with nano-1 (Fig. 2a). STS current-voltage (I-V) spectra were acquired by interrupting the feedback loop and the lateral scanning and recording the current as a function of the bias voltage. In order to reduce spectral noise, $20 \mathrm{I}-\mathrm{V}$ curves were signal averaged and, after numerical differentiation, the resulting $\mathrm{dI} / \mathrm{dV}$ curve was Fourier filtered. The tunneling conductance, $\mathrm{dI} / \mathrm{dV}$, which is proportional to the local density of states (DOS), of nano-1/SWNT dispersions exhibited peaks corresponding to van Hove singularities (Fig. 2b). These features clearly originate from the 1D SWNTs as the nano-1 DOS is featureless. Moreover, the $\mathrm{dI} / \mathrm{dV}$ spectra of nano-1/SWNT dispersions showed both vanishing and non-vanishing DOS in the vicinity of the Fermi energy, suggesting that the SWNTs coated with nano-1 are electronically semiconducting and metallic, respectively. Finally, STS dI/dV (DOS) spectra acquired from uncoated and peptide-coated regions of the same SWNT revealed that the DOS of the peptide-coated portion closely resembles that of the uncoated region with some slight modifications (Fig. 3).

\section{References}

[1] G. R. Dieckmann et al., J. Am. Chem. Soc. 125 (2003) 1770.

[2] V. Zorbas et al., J. Am. Chem. Soc. 126 (2004) 7222.

[3] The support of this research by the Robert A. Welch Foundation [AT-1326 (I.H.M.)] and Department of Homeland Security (V.Z.) is gratefully acknowledged. 

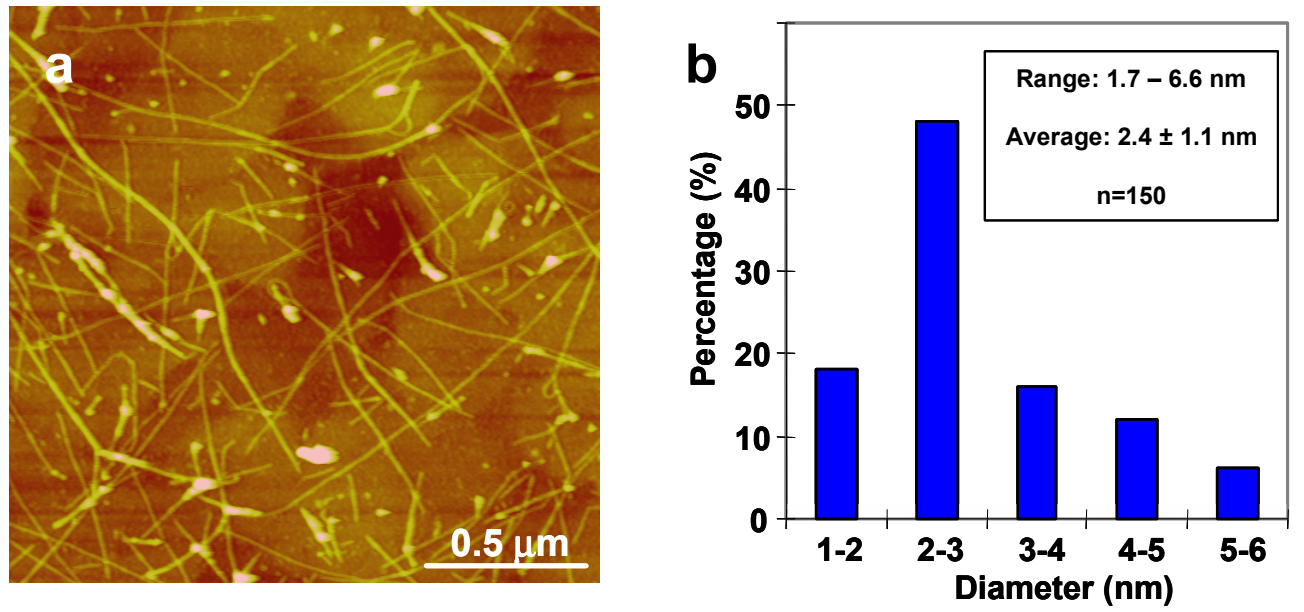

Figure 1. (a) AFM image and (b) diameter distribution of nano-1/SWNT dispersion on $\mathrm{Au}(111)$.
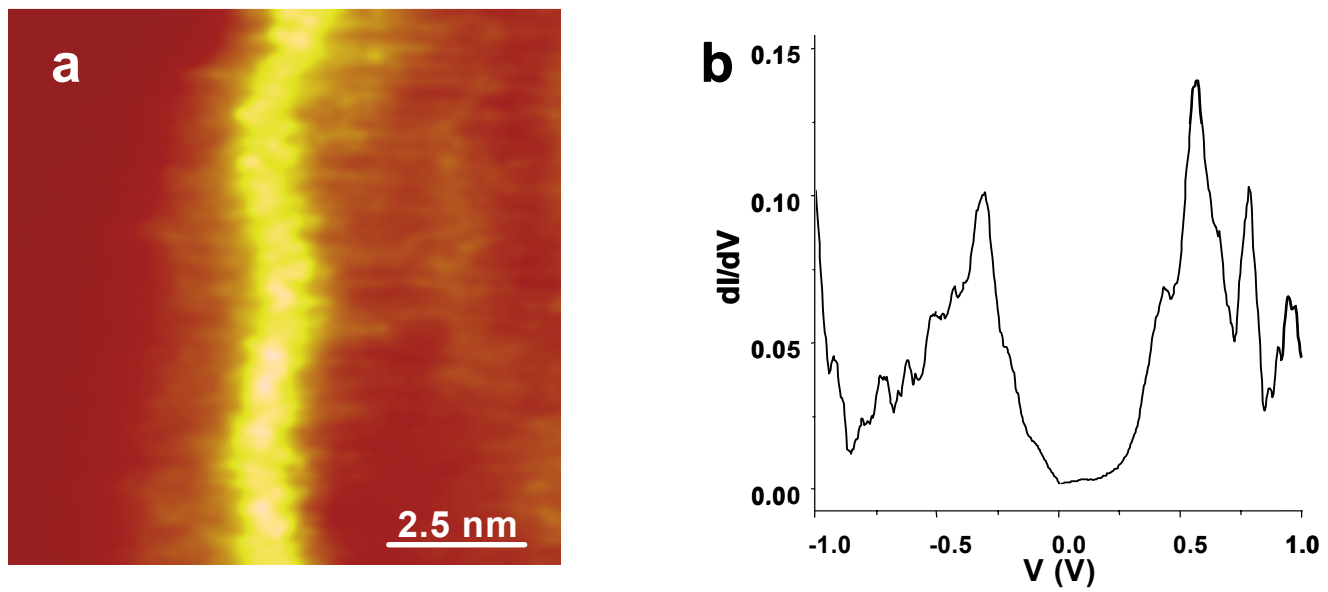

Figure 2. (a) STM image of nano-1/SWNT on Au(111), and (b) corresponding STS dI/dV spectra.
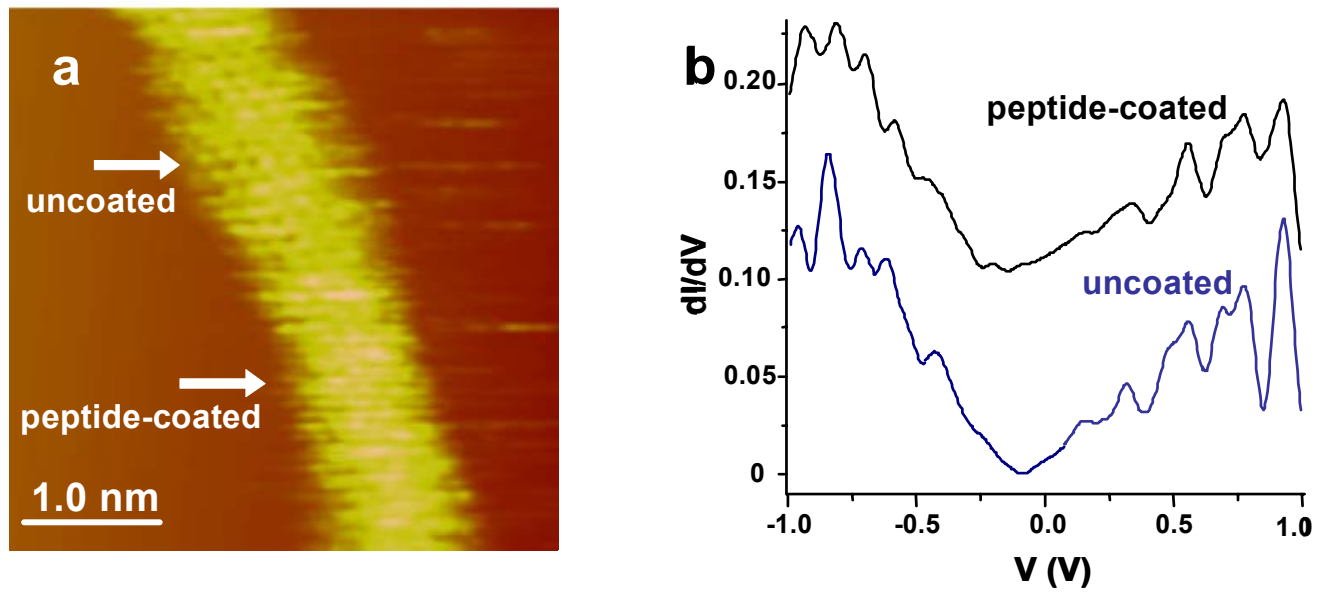

Figure 3. (a) STM image of nano-1/SWNT on $\mathrm{Au}(111)$, where arrows indicate uncoated and peptidecoated regions, and (b) corresponding STS dI/dV spectra (DOS) of the two regions, offset for clarity. 